\title{
Stagnation Hugoniot Analysis for Steady Combustion Waves in Propulsion Systems
}

\author{
E. Wintenberger* and J. E. Shepherd ${ }^{\dagger}$ \\ California Institute of Technology, Pasadena, California 91125
}

\begin{abstract}
The combustion mode in a steady-flow propulsion system has a strong influence on the overall efficiency of the system. To evaluate the relative merits of different modes, we propose that it is most appropriate to keep the upstream stagnation state fixed and the wave stationary within the combustor. Because of the variable wave speed and upstream stagnation state, the conventional Hugoniot analysis of combustion waves is inappropriate for this purpose. To remedy this situation, we propose a new formulation of the analysis of stationary combustion waves for a fixed initial stagnation state, which we call the stagnation Hugoniot. For a given stagnation enthalpy, we find that stationary detonation waves generate a higher entropy rise than deflagration waves. The combustion process generating the lowest entropy increment is found to be constant-pressure combustion. These results clearly demonstrate that the minimum entropy property of detonations derived from the conventional Hugoniot analysis does not imply superior performance in all propulsion systems. This finding reconciles previous analysis of flowpath performance analysis of detonation-based ramjets with the thermodynamic cycle analysis of detonation-based propulsion systems. We conclude that the thermodynamic analysis of propulsion systems based on stationary detonation waves must be formulated differently than for propagating waves, and the two situations lead to very different results.
\end{abstract}

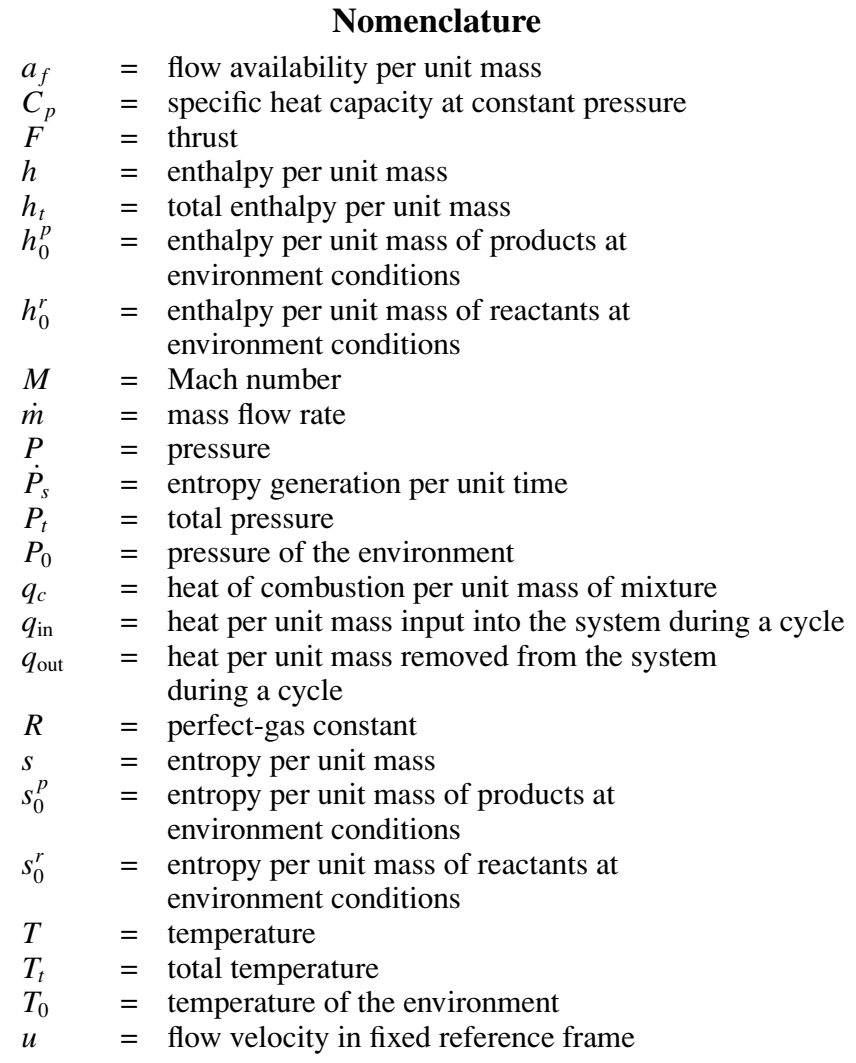

Presented as Paper 2004-1033 at the AIAA 42nd Aerospace Sciences Meeting and Exhibit, Reno, NV, 5 January 2004; received 10 August 2004; revision received 22 November 2005; accepted for publication 5 October 2005. Copyright (C) 2005 by California Institute of Technology. Published by the American Institute of Aeronautics and Astronautics, Inc., with permission. Copies of this paper may be made for personal or internal use, on condition that the copier pay the $\$ 10.00$ per-copy fee to the Copyright Clearance Center, Inc., 222 Rosewood Drive, Danvers, MA 01923; include the code 0748-4658/06 \$10.00 in correspondence with the CCC.

*Graduate Student, Graduate Aeronautical Laboratories; currently Thermal Engineer, GE Advanced Materials Quartz, Strongsville, Ohio 44149.

${ }^{\dagger}$ Professor, Aeronautics, Graduate Aeronautical Laboratories. Member AIAA.

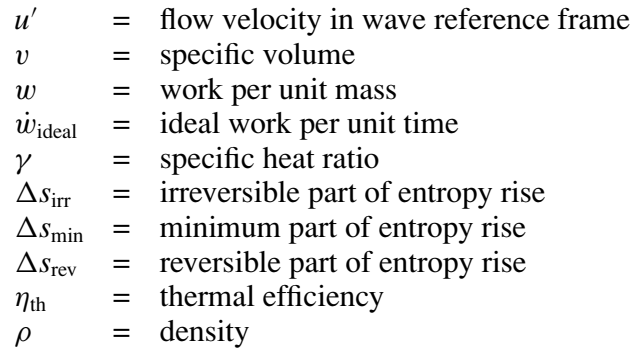

\section{Introduction}

KEY issue in conceptual design and analysis of proposed $A$ propulsion systems is the role of the combustion mode in determining the overall efficiency of the system. Because entropy production is detrimental to the efficiency of propulsion systems, ${ }^{1}$ optimizing a propulsion system means minimizing the overall entropy generation in the flow. The entropy increment associated with the combustion process is often the largest of all increments through the propulsion system. This is why the selection of the combustion mode is critical to engine performance.

Chapman-Jouguet (CJ) detonation waves are of particular interest because they apparently correspond to the minimum entropy generation $^{2}$ of all combustion wave modes. This result, along with the similarities between detonation and constant-volume combustion, has often been quoted in the literature as a motivation to explore detonation applications to propulsion. ${ }^{3-5}$ On a thermodynamic basis, idealized unsteady propulsion systems based on propagating detonations appear to have the potential for high efficiency. However, the situation appears to be the opposite for steady-flow propulsion systems based on stationary detonation waves in spite of the apparent lower entropy rise generated by detonations as compared with deflagrations. The performance ${ }^{6-8}$ of steady detonation-based engines is systematically and substantially lower than that of the deflagration-based engines.

These results are in agreement with the early work of Zel'dovich ${ }^{9}$ and $\mathrm{Foa}^{3,10}$ on steady-flow propulsion. Looking at a detonation wave as a shock wave followed by a reaction zone, Zel'dovich qualitatively argued that this process generates more entropy than a deflagration and estimated the thrust reduction associated with the use of a detonation wave instead of a deflagration in an airbreathing jet engine. ${ }^{9}$ Foa concluded ${ }^{10}$ that, in a constant-area burner, 
constant-pressure combustion is the optimal combustion mode using a polytropic representation of the combustion process. He also recognized ${ }^{3}$ that, in steady-flow propulsion systems, the type of combustion mode is constrained by the requirement for a stationary reaction front, and that combustion modes should be compared for a fixed stagnation temperature. On this basis, Foa compared a subsonic-combustion ramjet and a detonation-based ramjet and concluded that subsonic combustion produced a lower entropy rise than steady detonation. ${ }^{3}$

The differing results for steady-flow and propagating detonation propulsion system analysis lead us to reexamine the conventional Hugoniot analysis and the minimum entropy property of the CJ point. In doing so, it is important to distinguish between using propagating detonation waves in an unsteady cycle, as in a pulse detonation engine, and stationary waves in a steady cycle, as in a detonation ramjet or oblique detonation engine. Despite the common feature of detonation, the boundary conditions for the combustor are completely different for the two applications, and the nature of the cycle is completely different. In pulse detonation engines, an unsteady, cyclic thermodynamic analysis with a propagating detonation wave is appropriate. In a detonation ramjet, a steady open-system thermodynamic analysis with a stationary detonation wave is appropriate. In the present paper, we focus exclusively on the steady-flow case with a stationary wave oriented normal to the flow. Although our results could be extended in a straightforward way to stationary oblique waves, the main points of our investigation can most readily be seen for the simplest case of waves normal to the flow. The thermodynamic analysis for the unsteady case is discussed elsewhere. ${ }^{11}$ The present study is also restricted to considering idealized detonations that can be treated through the jump conditions and thermodynamics. We recognize that chemical kinetics, detonation-wave structure, and instability are important features of detonations, ${ }^{12}$ but, in the present study, we focus on the mean flow and thermodynamic aspects; some considerations about stability and wave structure are given by Wintenberger. ${ }^{13}$

We start our discussion by reviewing standard thermodynamic cyclic analysis, focusing on the role of entropy generation in determining efficiency. Next, we consider the conventional Hugoniot analysis and conclude that this approach is not appropriate for comparing combustion modes for stationary combustion waves in propulsion systems. We propose that in order to compare combustion modes on an equal basis in steady flow we need to fix the upstream stagnation state and require that the combustion wave speed be determined so that the wave is always stationary relative to the propulsion system. We reformulate the conventional analysis of steady combustion waves to obtain solutions for a fixed initial stagnation state and conclude that steady detonation waves generate more entropy than steady deflagrations at a given flight condition. In particular, focusing on the irreversible portion of the entropy rise in the combustion process, we demonstrate that it is the irreversibility associated with stationary detonation waves in steady flow, which causes the poor performance of steady detonation-based propulsion.

\section{Thermodynamic Cycle Analysis}

The thermodynamic processes encountered in airbreathing propulsion involve sequential compression, combustion, and expansion. This sequence is turned into a closed cycle through a constantpressure process during which the fluid exhausted into the atmosphere at the end of the expansion process is converted into the inlet fluid by exchanging heat and work with the surroundings. The thermal efficiency of an arbitrary cycle involving adiabatic combustion can be defined as the ratio of the work done by the system to the heat of combustion of the fuel-air mixture (on a specific mass basis).

$$
\eta_{\mathrm{th}}=w / q_{c}
$$

The meaning of work done and mixture heat of combustion can be clarified by considering a thermodynamic cycle consisting of an arbitrary adiabatic process taking the system from its initial state 1 to state 4 , and ending with a constant-pressure process taking the system back to state 1 . In the present case, the system consists of

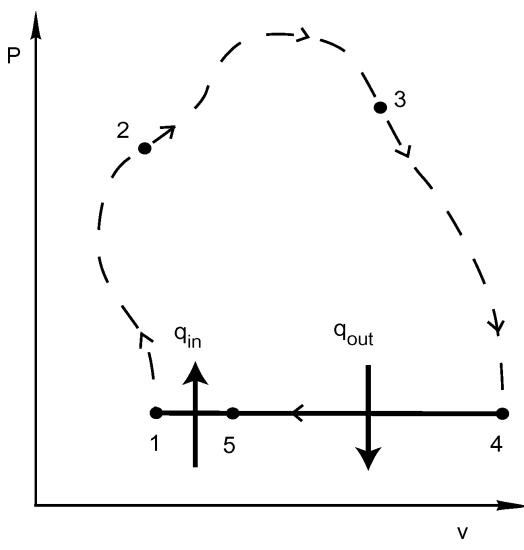

Fig. 1 Arbitrary thermodynamic cycle ending with constant-pressure process.

a fixed mass of working fluid. Between states 1 and 2, the working fluid is a fuel-air mixture. We suppose that an adiabatic combustion process transforms the working fluid from reactants to products between states 2 and 3. The working fluid is combustion products between states 3 and 5. The sequence of states 1-2-3-4 are shown with a dashed line because these processes can involve nonequilibrium states and processes that cannot be represented uniquely in the pressure-volume plane.

Because processes 1-2-3-4 are adiabatic, the heat interactions exist only between states $4-5-1$. We will suppose that states 4 and 5 are in chemical and thermal equilibrium and state 1 is a mixture of fuel and air that is in thermal but not chemical equilibrium. As shown in Fig. 1, there is an intermediate state 5 between 4 and 1 that divides the heat interaction into a heat removal (4-5) step and heat addition (5-1) step. The heat interaction between steps 4 and 5 is required to remove an amount of thermal energy $q_{\text {out }}>0$ from the products of combustion and cool the flow down from the exhaust temperature to the ambient conditions. Because this process occurs at constant pressure, the First Law of Thermodynamics determines the heat interaction from the enthalpy change:

$$
q_{\text {out }}=h_{4}-h_{5}
$$

The heat interaction between steps 5 and 1 is required to add an amount of thermal energy $q_{\text {in }}>0$ in order to convert the combustion products back to reactants. The First Law of Thermodynamics can again be used to compute the heat interaction from the enthalpy change:

$$
q_{\text {in }}=h_{1}-h_{5}
$$

Note that this defines the quantity $q_{c}=q_{\text {in }}$ in a fashion consistent with standard thermochemical practice if the ambient conditions correspond to the thermodynamic standard state. Applying the First Law of Thermodynamics around the cycle, the work done by the system can be computed as the net heat interaction

$$
w=q_{\text {in }}-q_{\text {out }}=h_{1}-h_{4}
$$

The thermal efficiency [Eq. (1)] can, therefore, be rewritten as

$$
\eta_{\mathrm{th}}=\left(h_{1}-h_{4}\right) /\left(h_{1}-h_{5}\right)=\left(h_{1}-h_{4}\right) / q_{c}
$$

For steady-flow engines, the cycle analysis based on a closed system (fixed mass of material) is completely equivalent to the flowpath analysis based on an open system, as long as the mass and momentum contributions of the fuel are negligible and the exhaust flow is fully expanded at the exit plane. ${ }^{3}$ Within these assumptions, we can make a correspondence between states in the cyclic process of Fig. 1 and an open thermodynamic cycle. If the states in the open and closed cycles are equivalent, then the thermal efficiencies are the same for the two processes. The precise equivalence is based on the control-volume analysis of the energy balance in an open system whose inlet plane is at state 1 and exit plane is at state 4 . For an 
adiabatic open system with no work interaction, the energy balance between the inlet and exit for a quasi-one-dimensional system ${ }^{14}$ yields

$$
h_{1}+u_{1}^{2} / 2=h_{4}+u_{4}^{2} / 2
$$

Using the cycle thermal efficiency as defined in Eq. (1), we find that

$$
\eta_{\mathrm{th}}=\left(u_{4}^{2}-u_{1}^{2}\right) / 2 q_{c}
$$

Based on this equivalence, the thrust of a steady pressurematched propulsion system can be directly calculated from the thermal efficiency. ${ }^{3}$

$$
F=\dot{m}_{1}\left(u_{4}-u_{1}\right)=\dot{m}_{1}\left(\sqrt{u_{1}^{2}+2 \eta_{\text {th }} q_{c}}-u_{1}\right)
$$

This is the key link between ideal steady-flow propulsion system performance and thermodynamic cycle analysis. For steady flows, thermodynamic analysis of an idealized cycle is used to compute the efficiency through Eq. (5), and then the thrust can be computed by equating this to the efficiency defined by Eq. (7).

For an ideal (reversible) process, the heat removed during the constant-pressure process $4-5$ can be expressed as

$$
q_{\text {out }}=\int_{s_{5}}^{s_{4}} T \mathrm{~d} s
$$

and the thermal efficiency is

$$
\eta_{\mathrm{th}}=1-\int_{s_{5}}^{s_{4}} T \mathrm{~d} s / q_{c}
$$

For a given initial state 1 and a given mixture, state 5 is fixed, and the value of the entropy is determined by the specific heat of combustion and the product and reactant composition. Thus, the heat removed $q_{\text {out }}$ increases and the thermal efficiency decreases with increasing values of $s_{4}$. In general, the thermal efficiency is maximized when the entropy rise during process $1-4$ is minimized.

This general result can be computed explicitly if we consider a perfect gas and take $s_{5}=s_{1}$, which is approximately satisfied for real mixtures and exactly so for the simple model discussed later in this paper. The integral of Eq. (9) is calculated explicitly as a function of the entropy rise between states 1 and 4, and the thermal efficiency becomes

$$
\eta_{\text {th }}=1-\left(C_{p} T_{1} / q_{c}\right)\left\{\exp \left[\left(s_{4}-s_{1}\right) / C_{p}\right]-1\right\}
$$

Another approach highlighting the role of entropy generation in a steady-flow system is to consider the flow availability. The flow availability per unit mass is defined relative to the environment at pressure $P_{0}$ and temperature $T_{0}$ as the maximum theoretical work obtainable as the flow is brought to equilibrium with the environment.

$$
a_{f}=h-h_{0}-T_{0}\left(s-s_{0}\right)+u^{2} / 2
$$

Note that the values of $h_{0}$ and $s_{0}$ are different for reactants and products. The availability balance between the inlet and exit planes of a single-stream, steady-flow, adiabatic system is

$$
\dot{m}\left(a_{f 4}-a_{f 1}\right)=\dot{w}_{\text {ideal }}-T_{0} \dot{P}_{s}
$$

where $\dot{w}_{\text {ideal }}=\dot{m}\left[h_{0}^{r}-h_{0}^{p}-T_{0}\left(s_{0}^{r}-s_{0}^{p}\right)\right]$ is the ideal work per unit time that can be obtained from the conversion of reactants into products at environment conditions. The Second Law of Thermodynamics states that the entropy generation per unit time $\dot{P}_{s}=$ $\dot{m}\left(s_{4}-s_{1}\right) \geq 0$. Thus, it is clear from Eq. (13) that the entropy generation between states 1 and 4 reduces the increase in flow availability from its maximum value and, therefore, the propulsive power. ${ }^{15}$

The overall entropy rise is the sum of the entropy rise generated by combustion and of the entropy increments generated by irreversible processes such as shocks, friction, heat transfer, Rayleigh losses (combustion or equivalent heat addition at finite Mach number), or fuel-air mixing. ${ }^{3}$ A portion of the entropy increment generated by the combustion process is associated with the fact that the temperature increases significantly in combustion, analogous to the entropy increase that is produced by a reversible addition of heat to a nonflowing system. However, this entropy increment also has an irreversible component, which depends on the combustion mode.

The thermodynamic cycle analysis of this section points to the key role of entropy generation in determining cycle efficiency and propulsion performance. In the following sections, we will focus on determining the entropy generation involved in various combustion modes. Once the entropy change in the combustion process is computed, we can use either Eq. (10) or (11) to determine the efficiency from the entropy change and Eq. (8) to find the propulsion system performance. Applications of this method to model systems involving detonation and deflagration combustion modes are given by Wintenberger and Shepherd ${ }^{8}$ and Wintenberger. ${ }^{13}$

\section{Entropy Variation Along the Hugoniot}

In this section, we supply the well-known and basic facts regarding the elementary gas dynamics and thermodynamics of detonation waves considered as discontinuities. The different steady combustion modes that can be obtained are usually analyzed using a control volume surrounding the combustion wave, such as that of Fig. 2. The mass, momentum, and energy conservation equations ${ }^{14}$ for an idealized, one-dimensional wave with no storage in the control volume and or diffusive transport at the control volume boundaries are

$$
\begin{aligned}
\rho_{1} u_{1}^{\prime} & =\rho_{2} u_{2}^{\prime} \\
P_{1}+\rho_{1} u_{1}^{\prime 2} & =P_{2}+\rho_{2} u_{2}^{\prime 2} \\
h_{1}+u_{1}^{\prime 2} / 2 & =h_{2}+u_{2}^{\prime 2} / 2
\end{aligned}
$$

States 1 and 2 correspond respectively to the reactants upstream of the wave and the products downstream of the wave. The upstream state composition is specified, and the downstream state composition is determined by requiring that the products are in chemical equilibrium. The equation of state for the products must also be specified to complete the description. For the cases we consider, the ideal-gas equation of state and tabulated thermodynamic properties are the appropriate level of description. The usual analysis considers fixed thermodynamic conditions upstream $\left(P_{1}, \rho_{1}, h_{1}\right)$ and a variable inflow velocity $u_{1}^{\prime}$. Although this is the conventional approach, as we will see later, it is not the most appropriate approach for optimizing steady, airbreathing propulsion systems.

The Hugoniot curve determines the locus of the possible solutions for state 2 from a given state 1 and a given energy release $q_{c}$. In particular, it is instructive to plot the Hugoniot on a pressurespecific volume diagram (Fig. 3). The dashed portion of the curve labeled "forbidden" is physically impossible for Rayleigh processes. ${ }^{2}$ The solutions located in the upper branch of the Hugoniot are supersonic waves (detonations), whereas the solutions located in the lower branch are subsonic waves (deflagrations).

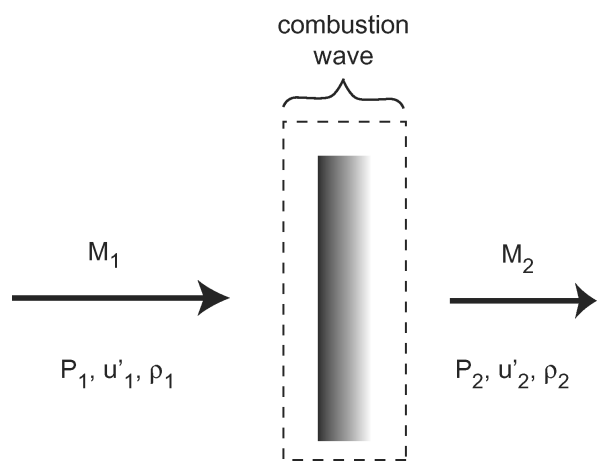

Fig. 2 Control volume used to analyze steady combustion waves. 


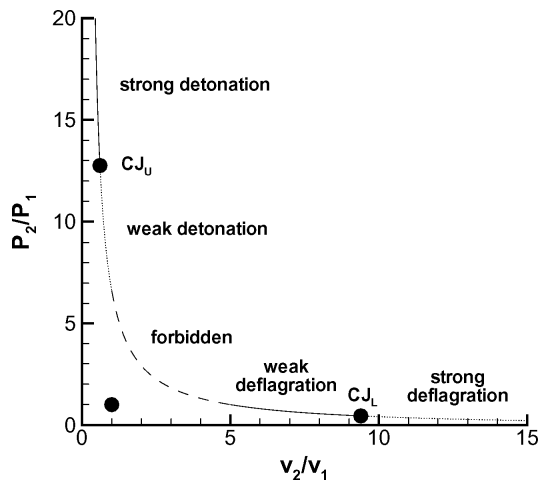

Fig. 3 Hugoniot in the pressure-specific volume plane for a perfect gas with $\gamma=1.4$ and $q_{c} / C_{p} T_{1}=4$.

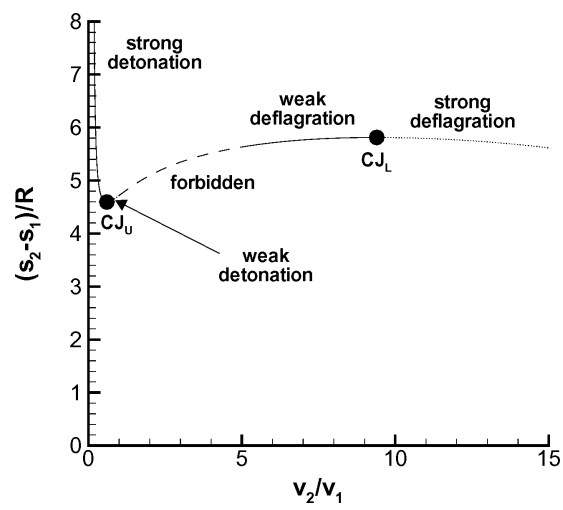

Fig. 4 Variation of the total entropy rise along the Hugoniot for a perfect gas with $\gamma=1.4, q_{c} / C_{p} T_{1}=4$.

The points where the Rayleigh line is tangent to the Hugoniot curve are called the CJ points. ${ }^{2}$ The CJ points are characterized by sonic flow downstream of the combustion wave and correspond to entropy extrema of the burned gases. It is possible to show, based on the curvature of the Hugoniot curve ${ }^{2}$ that the entropy is minimum at the upper $\mathrm{CJ}$ point and maximum at the lower $\mathrm{CJ}$ point (Fig. 4). The CJ points divide the possible locus of solutions into four regions, corresponding to strong detonations (supersonic flow to subsonic), weak detonations (supersonic to supersonic), weak deflagrations (subsonic to subsonic), and strong deflagrations (subsonic to supersonic). Strong deflagrations and weak detonations can be ruled out except in extraordinary situations by considering the reaction zone structure. ${ }^{2}$ The physically acceptable and observed solutions for steady waves are weak deflagrations and strong detonations. The solution to Eqs. (14-16) is uniquely determined only with some additional considerations. For deflagrations, the structure of the combustion wave and turbulent and diffusive transport processes determine the actual propagation speed. For detonations, gas dynamic considerations are apparently sufficient to determine the propagation speed (corresponding to the $\mathrm{CJ}_{U}$ solution), independent of the actual structure of the wave. ${ }^{2}$ Strong detonations are observed only in the transient state or if there is an "effective" piston created by the flow following the wave.

We now consider the case of the perfect gas $P=\rho R T$ in order to numerically illustrate the previous points. We will assume equal specific heat capacities for reactants and products

$$
C_{p}=[\gamma /(\gamma-1)] R
$$

and the enthalpy in the reactants and products can be expressed as

$$
h_{1}=C_{p} T_{1} \quad h_{2}=C_{p} T_{2}-q_{c}
$$

The set of Eqs. (14-16) can be rewritten for a perfect gas as a function of the Mach numbers upstream and downstream of the wave.

$$
\begin{gathered}
\frac{\rho_{2}}{\rho_{1}}=\frac{M_{1}^{2}\left(1+\gamma M_{2}^{2}\right)}{M_{2}^{2}\left(1+\gamma M_{1}^{2}\right)} \\
\frac{P_{2}}{P_{1}}=\frac{1+\gamma M_{1}^{2}}{1+\gamma M_{2}^{2}} \\
\frac{q_{c}}{C_{p} T_{1}}+1+\frac{\gamma-1}{2} M_{1}^{2}=\frac{M_{2}^{2}\left(1+\gamma M_{1}^{2}\right)^{2}}{M_{1}^{2}\left(1+\gamma M_{2}^{2}\right)^{2}}\left(1+\frac{\gamma-1}{2} M_{2}^{2}\right)
\end{gathered}
$$

This set of equations can be solved analytically for a given $q_{c}$ and initial state, and the resulting Hugoniot for the perfect gas is given in Fig. 3. Note that the solution curves shown in Figs. 3 and 4 are parameterized by $M_{1}$. Each point on the curve corresponds to a different combustion wave speed and requirements for combustor design.

The entropy rise associated with the combustion process can be computed from Eqs. (19) and (20).

$$
\left(s_{2}-s_{1}\right) / R=[\gamma /(\gamma-1)] \ln \left(T_{2} / T_{1}\right)-\ln \left(P_{2} / P_{1}\right)
$$

The entropy rise is plotted in Fig. 4 as a function of the specific volume. The different solution regions are shown, and the entropy rise has a global minimum at the $\mathrm{CJ}$ detonation point and a global maximum at the $\mathrm{CJ}$ deflagration point. Thus, from Eq. (11) it appears as if a cycle using detonation combustion will yield the highest thermal efficiency because it has the lowest entropy rise.

\section{Role of Irreversibility in Steady-Flow Propulsion}

The fact that the entropy rise is minimum at the CJ detonation point, in conjunction with the result of Eq. (10), has motivated several efforts to explore stationary detonation applications to steadyflow propulsion. ${ }^{6-8}$ However, these studies concluded that the performance of steady detonation-based engines is always substantially lower than that of the ramjet. The explanation of this apparent contradiction lies in considering the role of entropy generation and irreversible processes in the combustor. It is a general conclusion of thermodynamics and can be explicitly shown using availability arguments ${ }^{15}$ [Eq. (13)] that the work obtained is maximized when the irreversibility is minimized. When portions of the propulsion system involve losses and irreversible generation of entropy, the efficiency is reduced, and the reduction in performance (specific thrust) can be directly related to the irreversible entropy increase. ${ }^{1}$

The entropy rise occurring during premixed combustion in a flowing gas has a minimum component caused by the energy release and the chemical reactions, and an additional, irreversible, component caused by the finite velocity and, in the case of a detonation, the leading shock wave.

$$
s_{2}-s_{1}=\Delta s_{\min }+\Delta s_{\text {irr }}
$$

For a combustion wave such as that in Fig. 2, we propose that the minimum entropy rise (for a fixed upstream state and velocity) can be computed by considering the ideal stagnation or total state. (This conjecture is easy to demonstrate for a perfect gas with an effective heat-addition model of combustion. ${ }^{16}$ We also demonstrate the correctness of this idea explicitly in subsequent computations for the one- $\gamma$ detonation model and numerical solutions with realistic thermochemistry). The total properties at a point in the flow are defined as the values obtained by isentropically bringing the flow to rest. For example, the total enthalpy is

$$
h_{t}=h+u^{2} / 2
$$

and the total pressure and temperature are defined by

$$
h\left(P_{t}, s\right)=h_{t} \quad h\left(T_{t}, s\right)=h_{t}
$$




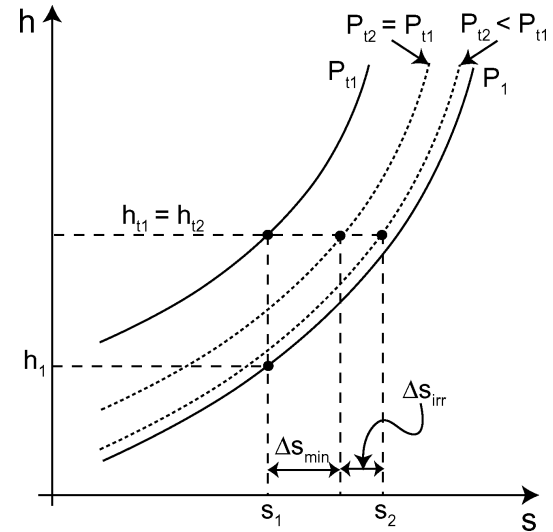

Fig. 5 Mollier diagram used to calculate minimum entropy component: - , isobars for reactants; and $\cdots$, isobars for products.

where by definition $s_{t}=s$. The process of computing the stagnation state is illustrated graphically in the $(h, s)$ or Mollier diagram of Fig. 5. Differentiating Eq. (25) yields

$$
\mathrm{d} h_{t}=T_{t} \mathrm{~d} s+\left(1 / \rho_{t}\right) \mathrm{d} P_{t}
$$

At fixed total enthalpy, the total pressure decreases with increasing entropy

$$
\mathrm{d} P_{t}=-\rho_{t} T_{t} \mathrm{~d} s
$$

so that the minimum entropy rise is associated with the highest total pressure, which is the upstream value $P_{t 1}$. This is illustrated graphically in Fig. 5, showing the additional entropy increment $\Delta s_{\text {irr }}$ associated with a total pressure decrement $P_{t 1}-P_{t 2}$.

For a given stagnation state, the minimum entropy rise can be determined for gas mixtures with realistic thermochemistry by considering an ideal constant-pressure (zero velocity) combustion process. The first step is to determine the total temperature in the products from the energy balance equation

$$
h_{2}\left(T_{t 2}\right)=h_{1}\left(T_{t 1}\right)
$$

where the species in state 2 are determined by carrying out a chemical equilibrium computation. The second step is to determine the entropy rise across the combustion wave by using the stagnation pressures, temperatures, and compositions to evaluate the entropy for reactants and products

$$
\Delta s_{\min }=s_{2}\left(T_{t 2}, P_{t 1}\right)-s_{1}\left(T_{t 1}, P_{t 1}\right)
$$

The total entropy jump across the wave is

$$
s_{2}-s_{1}=s_{2}\left(T_{2}, P_{2}\right)-s_{1}\left(T_{1}, P_{1}\right)
$$

where state 2 in the products is determined by solving the jump conditions. The irreversible component can then be computed by using Eq. (23).

For a perfect-gas model, the entropy change can be explicitly computed as

$$
s_{2}-s_{1}=C_{p} \ln \left(T_{t 2} / T_{t 1}\right)-R \ln \left(P_{t 2} / P_{t 1}\right)
$$

From Eq. (29) the minimum entropy rise is

$$
\Delta s_{\min }=C_{p} \ln \left(T_{t 2} / T_{t 1}\right)
$$

and the irreversible component is

$$
\Delta s_{\text {irr }}=-R \ln \left(P_{t 2} / P_{t 1}\right)
$$

The minimum component can be identified as the amount of entropy increase that would occur with an equivalent reversible addition of heat

$$
\mathrm{d} s=\mathrm{d} q / T
$$

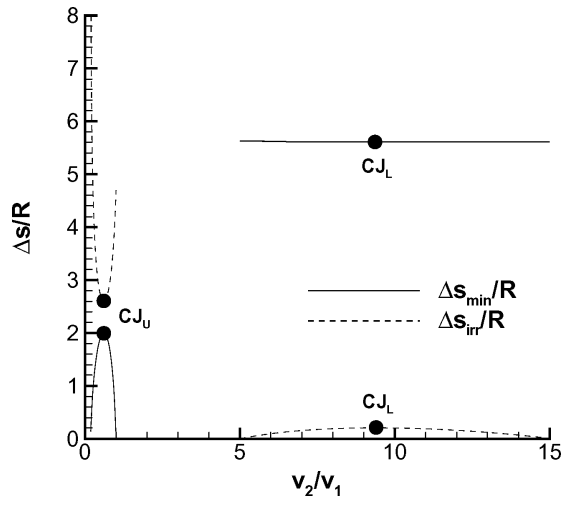

Fig. 6 Minimum and irreversible components of the entropy rise along the Hugoniot for a perfect gas with $\gamma=1.4$ and $q_{c} / C_{p} T_{1}=4$.

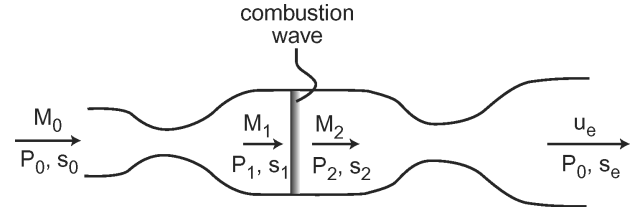

Fig. 7 Ideal steady engine in flight showing the location of the combustion wave.

at constant pressure, for which

$$
\mathrm{d} q=\mathrm{d} h=C_{p} \mathrm{~d} T
$$

Substituting and integrating from stagnation state 1 to 2 , we find that

$$
\Delta s_{\text {rev }}=C_{p} \ln \left(T_{t 2} / T_{t 1}\right)
$$

which is identical to the expression for the minimum entropy rise found from evaluating the entropy change using the prescription just given. Using these definitions, we show in Fig. 6 the partition of the entropy into these two portions for the perfect gas case considered earlier.

Although the total entropy rise is lower for the detonation branch than the deflagration branch, a much larger portion (greater than $50 \%$ ) of the entropy rise is irreversible for detonations than for deflagrations (less than 5\%). Separate computations show that the majority of the irreversible portion of the entropy rise for detonations is caused by the entropy jump across the shock front, which can be obtained directly from the total pressure decrease across the shock wave and Eq. (31). This loss in total pressure is orders of magnitude larger for detonation than for deflagration solutions and has been shown ${ }^{6}$ to be responsible for the lower performance of detonation-based engines relative to the ramjet. Hence, the paradox mentioned earlier can be resolved by considering not just the total entropy rise, but by determining what part of this is irreversible. An alternative way to look at this issue is given in the next section, where we reformulate the jump conditions so that the role of irreversible entropy rise in the calculation of the thermal efficiency can be demonstrated explicitly.

\section{Irreversible Entropy Rise and Thermal Efficiency}

The role of the irreversible part of the entropy rise can be explored further by considering Eq. (10). To compare objectively different combustion modes, the engine has to be studied in a given flight situation for a fixed amount of energy release during the combustion, as shown in Fig. 7. Our conceptual engine consists of an inlet, a combustion chamber with a steady combustion wave, and a nozzle. State 0 corresponds to the freestream conditions, whereas state 1 denotes the state of the flow just upstream of the combustion wave; state 2 is the state just downstream of the combustion wave, and state $e$ corresponds to the conditions at the exit plane of the engine. The conditions for combustion-wave stabilization are not considered in our thermodynamic analysis. We only require that the wave 
be stationary, that is, the flow Mach number just upstream of the combustion wave has to equal the wave propagation Mach number.

From a reference frame fixed relative to the engine, the boundary conditions upstream of the engine consist of air at fixed outside conditions (pressure $P_{0}$ and temperature $T_{0}$ ) flowing into the engine at a fixed velocity equal to the flight velocity $u_{0}$, as depicted in Fig. 7 . These boundary conditions correspond to a single value of the total enthalpy $h_{t 0}=h\left(T_{0}, P_{0}\right)+u_{0}^{2} / 2$ and of the entropy $s_{0}=s\left(T_{0}, P_{0}\right)$. Thus, they determine a single freestream stagnation state defined by the set of stagnation properties $h_{t 0}$ and $P_{t 0}=P\left(h_{t 0}, s_{0}\right)$. However, the static conditions just upstream of the combustion wave vary with the combustion mode and its associated propagation Mach number. Hence, fixed static conditions upstream of the combustion wave are not representative of actual flight situations, and the conventional Hugoniot analysis, which is based on this assumption, can be misleading when trying to compare the relative merits of various combustion modes. At a given flight condition, the static conditions ahead of a stationary wave will be different for different combustion modes, and the common factor will instead be the freestream stagnation state $\left(h_{t 0}, P_{t 0}\right)$.

The entropy rise between the inlet and exit planes is the sum of the entropy rise through the combustion and the irreversible entropy increments through the inlet and nozzle. Grouping together the irreversible entropy increments through the inlet, the combustion chamber, and the nozzle,

$$
s_{e}-s_{0}=\Delta s_{\min }+\Delta s_{\text {irr }}
$$

The minimum part of the entropy rise during combustion is constant for a fixed energy release and a fixed stagnation state upstream of the wave. From the general principles of thermodynamics and consistent with Eq. (10), the highest efficiency is obtained with the minimum irreversibility for a given chemical energy release $q_{c}$.

This general statement can be shown explicitly for the case of the perfect gas. Using the one- $\gamma$ model of detonations, ${ }^{14}$ the energy equation is expressed as

$$
C_{p} T_{t 2}=C_{p} T_{t 1}+q_{c}
$$

Combining Eq. (32) with the preceding expression, the minimum component of the entropy rise for the one- $\gamma$ model of detonations ${ }^{14}$ is

$$
\Delta s_{\min }=C_{p} \ln \left(1+q_{c} / C_{p} T_{t 1}\right)
$$

Substituting Eq. (37) into Eq. (11), and using the result of Eq. (39), the thermal efficiency can be expressed as a function of the irreversible entropy rise

$$
\eta_{\text {th }}=1-\left(C_{p} T_{0} / q_{c}\right)\left[\left(1+q_{c} / C_{p} T_{t 1}\right) \exp \left(\Delta s_{\text {irr }} / C_{p}\right)-1\right]
$$

Note that $T_{0}$ in this expression corresponds to $T_{1}$ used in Eq. (11) because in the present section we are using $T_{1}$ for the variable state just upstream of the wave. From Eq. (40), the highest efficiency is obtained for $\Delta s_{\text {irr }}=0$

$$
\eta_{\mathrm{th}}<\eta_{\mathrm{th}}\left(\Delta s_{\mathrm{irr}}=0\right)=1-T_{0} / T_{t 1}
$$

which is the classical expression for the ideal Brayton cycle.

Consider an idealized version of our conceptual engine, for which the thermal efficiency is determined only by the irreversible entropy rise during combustion. To compare different combustion modes, we need to calculate the irreversible entropy rise for all of the possible solutions to Eqs. (14-16). However, the result of Fig. 4 does not apply directly because the velocity of the initial state and, consequently, the total enthalpy are not constant for the conventional Hugoniot analysis. Instead, it is necessary to compute another solution curve corresponding to a fixed freestream stagnation state, which we will refer to as the stagnation Hugoniot.

\section{Stagnation Hugoniot}

The stagnation Hugoniot is the locus of the solutions to the conservation equations (14-16) for a given stagnation state upstream of the combustion wave. The stagnation Hugoniot analysis considers fixed total enthalpy $h_{t 1}$ and entropy $s_{1}$. The flow properties upstream of the combustion wave vary with inflow velocity $u_{1}^{\prime}$.

$$
\begin{gathered}
h_{1}=h_{t 1}-u_{1}^{\prime 2} / 2 \\
P_{1}=P\left(h_{1}, s_{1}\right) \\
v_{1}=v\left(h_{1}, s_{1}\right)
\end{gathered}
$$

All of the solution states to the stagnation Hugoniot can be found by varying the inflow velocity. Varying the inflow velocity modifies the state 1 just upstream of the combustion wave. We will refer to the local Hugoniot analysis as the conventional Hugoniot analysis for a given constant state 1 defined by a value of the inflow velocity along the stagnation Hugoniot. The general properties of the stagnation Hugoniot can be deduced from the classical results for the conventional Hugoniot. $^{2}$

Like in the conventional Hugoniot analysis, the inflow velocity has a minimum value along the detonation branch and a maximum value along the deflagration branch (Fig. 8). We show that these points correspond to the $\mathrm{CJ}$ conditions. The inflow velocity is related to the pressure and volume across the combustion wave through the Rayleigh line relationship.

$$
u_{1}^{\prime 2}=-v_{1}^{2} \cdot\left(P_{2}-P_{1}\right) /\left(v_{2}-v_{1}\right)
$$

Differentiating this equation, and keeping in mind that the properties at state 1 also vary,

$$
\begin{aligned}
\mathrm{d}{u_{1}^{\prime}}^{2} & =-\left[v_{1}^{2} /\left(v_{2}-v_{1}\right)^{2}\right]\left[\left(2 v_{2} / v_{1}-1\right)\left(P_{2}-P_{1}\right) \mathrm{d} v_{1}\right. \\
& \left.-\left(v_{2}-v_{1}\right) \mathrm{d} P_{1}+\left(v_{2}-v_{1}\right) \mathrm{d} P_{2}-\left(P_{2}-P_{1}\right) \mathrm{d} v_{2}\right]
\end{aligned}
$$

At the inflow velocity extrema (i.e., $\mathrm{d} u_{1}^{\prime}=0$ ), we have from Eq. (42) that $\mathrm{d} h_{1}=-u_{1}^{\prime} \mathrm{d} u_{1}^{\prime}=0$ and because the entropy at state 1 is given, $\mathrm{d} P_{1}=\mathrm{d} h_{1} / v_{1}=0$ and $\mathrm{d} v_{1}=0$. Thus, these points also correspond to extrema of enthalpy, pressure, and specific volume at state 1 . Simplifying all of the differentials vanishing at the inflow velocity extrema, Eq. (46) holds only if

$$
\frac{\partial P_{2}}{\partial v_{2}}=\frac{P_{2}-P_{1}}{v_{2}-v_{1}}
$$

which means that, for the upstream states corresponding to inflow velocity extrema, the local Hugoniot and the Rayleigh line are tangent. Thus, the points at which the inflow velocity has an extremum are $\mathrm{CJ}$ points.

From the conventional Hugoniot analysis, we know that the entropy along the local Hugoniot has an extremum at the CJ points, ${ }^{2}$

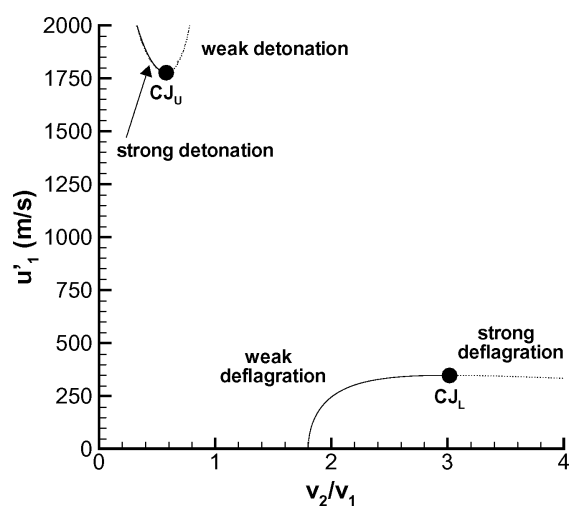

Fig. 8 Inflow velocity along the stagnation Hugoniot for a stoichiometric propane-air mixture: $P_{t 1}=100 \mathrm{~atm}, T_{t 1}=2000 \mathrm{~K}$, and $h_{t 1}=$ $2.113 \mathrm{MJ} / \mathrm{kg}$. 
that is, $\partial s_{2} / \partial v_{2}=0$. This result is straightforward to extend to the stagnation Hugoniot.

$$
\frac{\partial s_{2}}{\partial u_{1}^{\prime}}=\frac{\partial s_{2}}{\partial v_{2}} \cdot \frac{\partial v_{2}}{\partial u_{1}^{\prime}}=0
$$

Additionally, it can be shown that

$$
\frac{\partial^{2} s_{2}}{\partial u_{1}^{\prime 2}}=\frac{\partial^{2} s_{2}}{\partial v_{2}^{2}}\left(\frac{\partial v_{2}}{\partial u_{1}^{\prime}}\right)^{2}
$$

Based on the local Hugoniot analysis, ${ }^{2}$ we know that $\partial^{2} s_{2} / \partial v_{2}^{2} \geq 0$ at the $\mathrm{CJ}$ detonation point. Thus, the entropy along the stagnation Hugoniot is minimum at the CJ detonation point, and, conversely, maximum at the $\mathrm{CJ}$ deflagration point. Because the detonation and deflagration branches are disjoint, as we will see next, these are only local extrema. We now calculate the stagnation Hugoniot for the perfect gas case and carry numerical solutions for a case with realistic thermochemistry.

\section{Stagnation Hugoniot for the Perfect Gas}

We compute the stagnation Hugoniot for a perfect gas, based on Eqs. (19-21). Equation (21) has to be rewritten as a function of the parameter $q_{c} / C_{p} T_{t 1}$, which has a fixed value for a given freestream condition.

$$
1+\frac{q_{c}}{C_{p} T_{t 1}}=\frac{M_{2}^{2}\left(1+\gamma M_{1}^{2}\right)^{2}\left\{1+[(\gamma-1) / 2] M_{2}^{2}\right\}}{M_{1}^{2}\left(1+\gamma M_{2}^{2}\right)^{2}\left\{1+[(\gamma-1) / 2] M_{1}^{2}\right\}}
$$

This equation can be solved analytically for $M_{2}$ as a function of $M_{1}$. The solution curves shown in Figs. 9 and 10 can be obtained

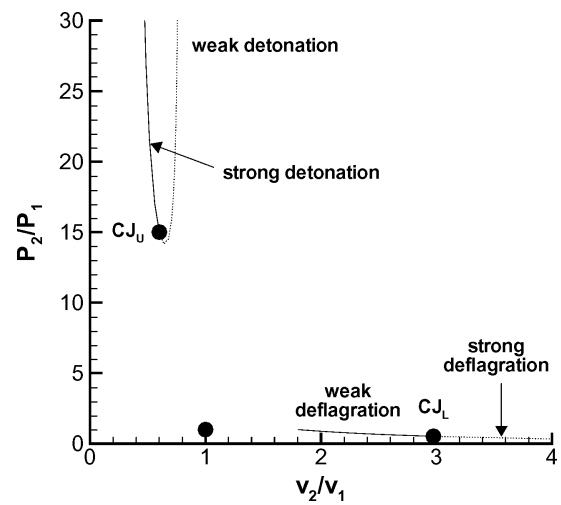

Fig. 9 Stagnation Hugoniot in the pressure-specific volume plane for a perfect gas with $\gamma=1.4$ and $q_{c} / C_{p} T_{t 1}=0.8$.

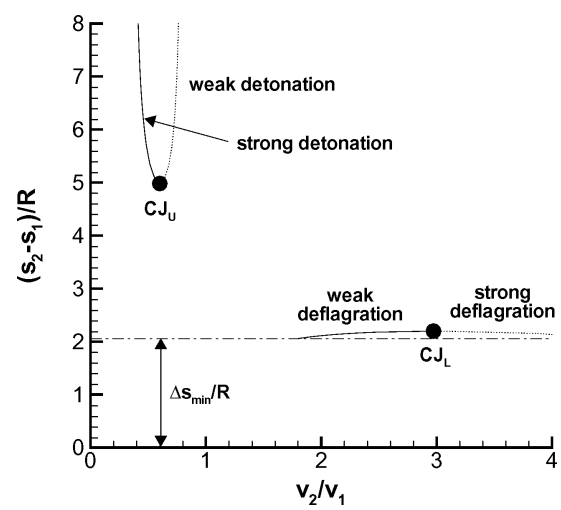

Fig. 10 Total entropy rise along the stagnation Hugoniot for a perfect gas. The minimum component of the entropy rise is fixed along the stagnation Hugoniot and is shown as the straight line. The total entropy variation is caused by the irreversible component only: $\gamma=1.4$ and $q_{c} / C_{p} T_{t 1}=0.8$. by parametric ( $M_{1}$ being the parameter) evaluation of Eqs. (19-21) or else as shown in the appendix, as explicit solutions in terms of $v_{2} / v_{1}$. Detonation solutions are found to be possible only for

$$
q_{c} / C_{p} T_{t 1}<1 /\left(\gamma^{2}-1\right)
$$

This condition is imposed by the requirement that $T_{1}>0$ and is in agreement with our results on detonation ramjets ${ }^{8}$ using a simple one- $\gamma$ detonation model. ${ }^{14}$ For higher values of $q_{c} / C_{p} T_{t 1}$, the total enthalpy is not high enough to enable a steady detonation in the combustor for the given value of the heat release, and no steady solutions exist.

For the conventional Hugoniot (Fig. 3), the entropy, pressure, and temperature at state 2 are finite for a constant-volume $\left(v_{2}=v_{1}\right)$ explosion process even though, in this limit, $M_{1} \rightarrow \infty$. However, in the stagnation Hugoniot representation the pressure ratio along the weak detonation branch becomes infinite as this limit is approached. As $M_{1} \rightarrow \infty, M_{2}$ asymptotes to a constant value instead of becoming infinite as for the conventional Hugoniot.

$$
M_{2} \rightarrow \sqrt{\frac{1-(\gamma-1)\left(q_{c} / C_{p} T_{t 1}\right)+\sqrt{1-\left(\gamma^{2}-1\right)\left(q_{c} / C_{p} T_{t 1}\right)}}{\gamma(\gamma-1)\left(q_{c} / C_{p} T_{t 1}\right)}}
$$

This is because the stagnation conditions at state 2 are fixed by the stagnation conditions at state 1 and the heat release. As $M_{1} \rightarrow \infty$, the static pressure at state 1 decreases towards zero because the total pressure is fixed, but the static pressure at state 2 remains finite as a result of the finite value of $M_{2}$. This explains the unusual shape of the stagnation Hugoniot, which is plotted in the pressurespecific volume plane for $\gamma=1.4$ and $q_{c} / C_{p} T_{t 1}=0.8$ in Fig. 9. Just as for the conventional Hugoniot, there is no solution in the positive quadrant of the pressure-specific volume plane for Rayleigh processes. However, unlike the conventional Hugoniot, the stagnation Hugoniot curve is not continuous across this forbidden region. This means that the detonation and deflagration branches are disjoint.

The total entropy rise along the stagnation Hugoniot is shown in Fig. 10 as a function of the specific volume ratio. For a fixed heat release and initial stagnation state, the minimum entropy rise is constant [Eq. (39)], and the variation of the total entropy rise is caused by the change in irreversible entropy rise associated with the combustion mode. As in the conventional Hugoniot, the CJ points correspond to extrema of the entropy. However, they are only local extrema because of the discontinuity of the solution curve in the pressure-specific volume plane. The $\mathrm{CJ}$ detonation point corresponds to a minimum in entropy along the detonation branch, while the CJ deflagration point corresponds to a maximum in entropy along the deflagration branch. However, the entropy rise associated with the CJ detonation point is much larger than that associated with the CJ deflagration point for all possible values of $q_{c} / C_{p} T_{t 1}$. In general, the irreversible entropy rise associated with any physical solution on the deflagration branch is much lower than that for any detonation solution. Of all physically possible steady combustion modes, constant-pressure (CP) combustion at zero Mach number is the process with the smallest entropy rise for a fixed stagnation condition.

The result of Fig. 10 shows explicitly that steady combustion waves with a finite (nonzero) velocity generate irreversible entropy. For deflagrations, this irreversible entropy rise is caused by combustion at finite velocity and is responsible for the total pressure losses classically observed for steady combustion at finite Mach number, ${ }^{16}$ for example, in ramjets. This effect is also present for detonations, but the presence of the shock wave in detonations contributes to the majority of the irreversible entropy rise.

\section{Stagnation Hugoniot for a Gas with Realistic Thermochemistry}

We carried out numerical computations to verify that the results obtained for the perfect-gas case extend to the case of a gas with realistic thermochemistry. We developed an numerical procedure using Stanjan ${ }^{17}$ to compute the stagnation Hugoniot. The procedure iterates on the wave inflow velocity $u_{1}^{\prime}$. For a given wave inflow velocity, 


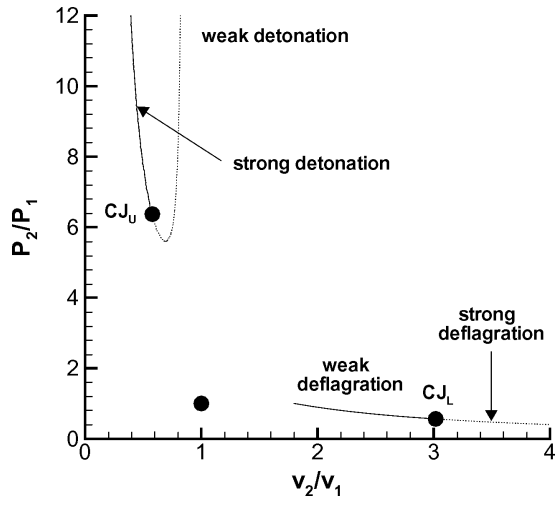

Fig. 11 Stagnation Hugoniot for a stoichiometric propane-air mixture: $P_{t 1}=100 \mathrm{~atm}, T_{t 1}=2000 \mathrm{~K}$, and $h_{t 1}=2.113 \mathrm{MJ} / \mathrm{kg}$.

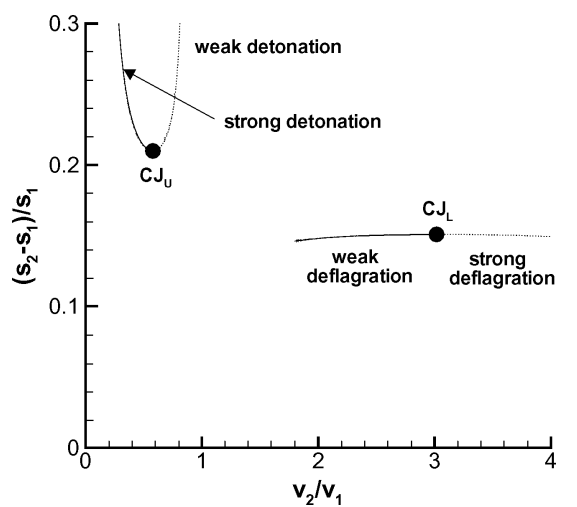

Fig. 12 Total entropy rise along the stagnation Hugoniot for a stoichiometric propane-air mixture: $P_{t 1}=100 \mathrm{~atm}, T_{t 1}=2000 \mathrm{~K}, h_{t 1}=$ $2.113 \mathrm{MJ} / \mathrm{kg}$, and $s_{1}=7.967 \mathrm{~kJ} / \mathrm{kgK}$.

Stanjan is used to compute a constant-entropy, frozen-composition solution from the stagnation conditions to state 1, whose enthalpy is determined by Eq. (42). Once state 1 is determined, Stanjan is used to compute the equilibrium solutions to the jump conditions [Eqs. (14-16)]. The procedure consists of using trial values of specific volume for detonation cases and the pressure for deflagration cases and calculating the other variable using Eq. (45). Based on the trial values upstream of the wave, Stanjan solves for the downstream equilibrium state using a specified pressure, specific volume equilibrium option. The enthalpy obtained from the equilibrium solution is then compared with the enthalpy calculated from the jump conditions [Eq. (16)], and the process is iterated until these two values are within a specified tolerance. Convergence is obtained using the bisection method.

The stagnation Hugoniot is plotted in the pressure-specific volume plane of Fig. 11 for a stoichiometric propane-air mixture. The stagnation conditions considered were chosen so that detonation solutions exist [see Eq. (51)]. The solution curve is similar to that obtained in the perfect-gas case and has two disjoint branches corresponding to deflagration and detonation solutions. The total entropy increment, normalized with the initial entropy value, is shown in Fig. 12 as a function of the specific volume ratio. The entropy rise has a local maximum at the $\mathrm{CJ}$ deflagration point and a local minimum at the $\mathrm{CJ}$ detonation point, but the increment associated with this local minimum is about $40 \%$ higher than the value corresponding to the local maximum. Thus, we find that, in the case of a gas with realistic thermochemistry, steady detonation waves generate more entropy than steady deflagrations. We also find that the minimum entropy rise for physically possible solutions is generated by constant-pressure combustion.

\section{Application to Steady-Flow Propulsion Systems}

We now use the result of Eq. (10) to compare the thermal efficiency of ideal steady propulsion systems as a function of the

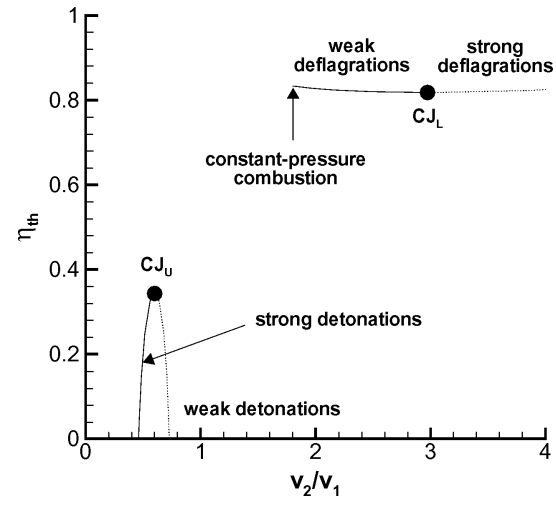

Fig. 13 Thermal efficiency of an ideal engine flying at $M_{0}=5$ as a function of the combustion mode selected for a perfect gas: $\gamma=1.4$ and $q_{c} / C_{p} T_{t 1}=0.8$.

combustion mode selected. Losses associated with shock waves, friction, mixing, or heat transfer are neglected, and the compression and expansion processes are assumed to be isentropic. The thermal efficiency for an ideal steady propulsion system flying at a Mach number of 5 is plotted in Fig. 13 for a perfect gas. The irreversible entropy rise in detonations strongly penalizes the efficiency of ideal steady detonation-based engines compared to the conventional ideal ramjet. Thus, this approach reconciles flowpath analysis and thermodynamic cycle analysis for detonation-based ramjets. Note that our thermodynamic cycle analysis yields identical results to the flowpath performance analysis of an ideal hydrocarbon-fueled detonation ramjet. ${ }^{8}$ However, the values of the thermal efficiency of Fig. 13 are not representative of practical propulsion systems at a flight Mach number $M_{0}=5$ because the total temperature at the combustor outlet is too high to be sustained by the chamber walls. More realistic studies limit the total temperature at the combustor outlet based on material considerations ${ }^{8}$ and account for additional losses, whose net effect is to significantly decrease the thermal efficiency. ${ }^{6}$

For our ideal propulsion system, the $\mathrm{CP}$ combustion process yields the highest thermal efficiency of all physical solutions to the conservation equations. Foa ${ }^{10}$ concluded that $\mathrm{CP}$ combustion was always the optimum solution for steady flow using an argument based on a polytropic approximation of the combustion mode for the perfect gas. We have now extended this result to all physically possible steady combustion modes for the perfect gas and the case of a gas with realistic thermochemistry.

However, in order to compare practical propulsion systems based on different combustion modes, one also has to compute the irreversible entropy rise through the other components of the engine. The entropy rise associated with other irreversible processes such as shocks, friction, mixing, or heat transfer can become significant ${ }^{1}$ and dominate the results, particularly at high supersonic flight Mach numbers. These points are important to consider when attempting to compare the merits of a detonation ramjet with a conventional ramjet. In particular, it is not obvious how a detonation ramjet flying near the CJ Mach number (with a minimal entropy rise through the inlet) would compare with a practical ramjet handicapped by a substantial entropy rise caused by the flow deceleration from supersonic to low subsonic velocities through the inlet. However, supersonic mixing in a detonation ramjet will entail an additional entropy rise much larger than that generated by subsonic mixing in the ramjet. Furthermore, the detonation ramjet is limited by condensation and autoignition of the fuel-air mixture. ${ }^{8}$ The issues of detonationwave stabilization and its stability in the combustor are still open questions,${ }^{8}$ and one can speculate that the detonation ramjet might suffer from problems similar to those associated with combustion instabilities in the ramjet.

\section{Conclusions}

We have used thermodynamic considerations to investigate the choice of the combustion mode for steady-flow propulsion 
and its consequence on propulsive performance. We reached the following conclusions:

1) The conventional Hugoniot analysis alone is inappropriate for analyzing the relative merits of steady combustion modes for propulsion.

2) For a given flight condition, the minimum entropy rise associated with combustion is fixed, and it is the irreversible component of the overall entropy rise in the system that determines the performance.

3) We reformulated the conventional analysis of steady combustion waves to obtain solutions for a fixed initial stagnation state. We propose that the stagnation Hugoniot analysis is the appropriate method for comparing combustion modes for a given flight condition.

4) For a given upstream stagnation state, steady detonation waves generate a higher entropy rise along the stagnation Hugoniot than deflagration waves, which makes them less desirable for propulsion applications. These findings reconcile thermodynamic cycle analysis with flowpath performance analysis of detonation-based ramjets. ${ }^{6-8}$

5) For a given upstream stagnation state, the combustion process generating the lowest entropy increment on the stagnation Hugoniot is constant-pressure combustion, corresponding to the ideal Brayton cycle.

This paper provides a framework under which all steady combustion modes can be directly compared for propulsion applications. To determine the overall performance of a system, one has to perform a detailed engineering analysis taking into account the entropy increments associated with combustion, shocks, mixing, viscous effects, and heat transfer. Our analysis singles out the entropy component associated with the combustion wave and can be used to estimate the contribution of the combustion process to the propulsive performance.

\section{Appendix: Explicit Solutions for the Perfect-Gas Model}

Solutions for the stagnation Hugoniot that are explicit in specific volume can be obtained by algebraic manipulation of the conservation relations [Eqs. (14-16)] in the case of a perfect gas with constant specific heats and a fixed heat release as specified in Eqs. (17) and (18). This model cannot accurately approximate both reactants and products with common values of $\gamma$ and $R$, but is often used to illustrate the qualitative behavior of real gases. In this spirit, we have provided explicit solutions for this model. We emphasize that for the case of propane-air mixtures (Figs. 8, 11, and 12) we have not relied on these solutions but have used numerical solutions of the conservation relations with realistic thermochemical states and species to compute equilibrium in the products.

The perfect-gas version of the velocity-volume relationship shown graphically in Fig. 8 is analytically expressed as

$$
\frac{u_{1}^{\prime 2}}{2 C_{p} T_{t 1}}=\left(1+\frac{q_{c}}{2 C_{p} T_{t 1}}-\frac{v_{2}}{v_{1}}\right) / \frac{\gamma+1}{\gamma-1}\left(1-\frac{v_{2}}{v_{1}}\right) \frac{v_{2}}{v_{1}}
$$

There are some limitations on the values that the specific volume ratio can assume because of the physical constraints on the conditions upstream of the wave. The temperature upstream of the wave is given by the stagnation enthalpy relationship [Eq. (42)] as

$$
T_{1} / T_{t 1}=1-u_{1}^{\prime 2} / 2 C_{p} T_{t 1}
$$

The temperature must be positive, and by definition the kinetic energy of the flow must also be positive so that

$$
0 \leq u_{1}^{\prime} / \sqrt{2 C_{p} T_{t 1}} \leq 1
$$

which limits the range of $v_{2} / v_{1}$ to the following:

Detonation branch:

$$
0<\left(v_{2} / v_{1}\right)_{1} \leq v_{2} / v_{1} \leq\left(v_{2} / v_{1}\right)_{2}<1
$$

Deflagration branch:

$$
1<\left(v_{2} / v_{1}\right)_{3} \leq v_{2} / v_{1}<\infty
$$

Table A1 Numerical values corresponding to critical volume ratios for perfect-gas stagnation Hugoniot with $\gamma=1.4$ and $q_{c} / C_{p 1} T_{t 1}=0.8$

\begin{tabular}{lc}
\hline \hline Volume ratio & Value \\
\hline$\left(v_{2} / v_{1}\right)_{1}$ & 0.3826 \\
$\left(v_{2} / v_{1}\right)_{\mathrm{CJ}, U}$ & 0.6000 \\
$\left(v_{2} / v_{1}\right)_{2}$ & 0.7840 \\
$\left(v_{2} / v_{1}\right)_{3}$ & 1.8000 \\
$\left(v_{2} / v_{1}\right)_{\mathrm{CJ}, L}$ & 3.000 \\
\hline \hline
\end{tabular}

The limiting volume ratios $\left(v_{2} / v_{1}\right)_{1}$ and $\left(v_{2} / v_{1}\right)_{2}$ on the detonation branch can be found by setting the velocity in Eq. (A1) to the maximum value $u_{1}^{\prime}=\sqrt{ }\left(2 C_{p} T_{t 1}\right)$. Solving the resulting quadratic equation, we find that

$$
\left(\frac{v_{2}}{v_{1}}\right)_{2}=\frac{\gamma}{\gamma+1} \mp \sqrt{\left(\frac{\gamma}{\gamma+1}\right)^{2}-\frac{\gamma-1}{\gamma+1}\left(1+\frac{q_{c}}{C_{p} T_{t 1}}\right)}
$$

The lower limit on the deflagration branch $\left(v_{2} / v_{1}\right)_{3}$ can be found by setting $u_{1}^{\prime}=0$.

$$
\left(v_{2} / v_{1}\right)_{3}=1+q_{c} / C_{p} T_{t 1}
$$

The CJ points can also be determined by finding the minimum (detonation branch) and maximum (deflagration branch) velocity points on each branch of the solution. The location of the CJ points is given by

$$
\left(\frac{v_{2}}{v_{1}}\right)_{U}=\left(1+\frac{q_{c}}{C_{p} T_{t 1}}\right) \mp \sqrt{\left(1+\frac{q_{c}}{C_{p} T_{t 1}}\right)^{2}-\left(1+\frac{q_{c}}{C_{p} T_{t 1}}\right)}
$$

where $U$ indicates the upper (detonation) and $L$ is the lower (deflagration) CJ point. For the case shown in Figs. 9 and 10, the computed values of the critical volume ratios are given in Table A1.

The pressure-volume relationship shown in Fig. 9 can be obtained as an analytic expression by substituting the velocity-volume relationship just given into the analog of the Rayleigh line (resulting from combining momentum and mass conservation) to obtain

$$
\begin{aligned}
\frac{P_{2}}{P_{1}} & =1+\frac{2 \gamma}{\gamma-1}\left(1-\frac{v_{2}}{v_{1}}\right)\left\{\left(1+\frac{q_{c}}{C_{p} T_{t 1}}\right.\right. \\
& \left.\left.-\frac{v_{2}}{v_{1}}\right) /\left[\frac{\gamma+1}{\gamma-1}\left(1-\frac{v_{2}}{v_{1}}\right) \frac{v_{2}}{v_{1}}+\frac{v_{2}}{v_{1}}-\left(1+\frac{q_{c}}{C_{p} T_{t 1}}\right)\right]\right\}
\end{aligned}
$$

This is the analog of the usual detonation Hugoniot or detonation adiabat.

The entropy-volume relationship shown in Fig. 10 can be obtained as an analytic expression from Eq. (22), where the pressure ratio is given by Eq. (A9) and the temperature ratio can be computed explicitly as a function of volume from $T_{2} / T_{1}=P_{2} / P_{1} \cdot v_{2} / v_{1}$. The entropy change predicted by this relationship actually becomes negative on the deflagration branch at sufficiently large specific volumes, $v_{2} / v_{1}>\left(v_{2} / v_{1}\right)_{\max } \approx 9.25$ for the case shown in Fig. 10. This results in a further limitation on the admissible values of $v_{2} / v_{1}$. As mentioned earlier, deflagration solutions with $v_{2} / v_{1}>\left(v_{2} / v_{1}\right)_{\mathrm{CJ}, L}$ and detonation solutions with $v_{2} / v_{1}>\left(v_{2} / v_{1}\right)_{\mathrm{CJ}, U}$ are considered ${ }^{2}$ nonphysical in nature. For this reason, the physically acceptable ranges of volumes $v_{2} / v_{1}$ in the stagnation Hugoniot analysis are smaller than given by Eqs. (A4) and (A5) and are restricted to the following:

Detonation branch:

$$
\left(v_{2} / v_{1}\right)_{1} \leq v_{2} / v_{1} \leq\left(v_{2} / v_{1}\right)_{\mathrm{CJ}, U}
$$

Deflagration branch:

$$
\left(v_{2} / v_{1}\right)_{3} \leq v_{2} / v_{1}<\left(v_{2} / v_{1}\right)_{\mathrm{CJ}, L}
$$




\section{Acknowledgments}

This work was supported by Stanford University Contract PY1905 under Department of Navy Grant N00014-02-1-0589 Pulse Detonation Engines: Initiation, Propagation, and Performance. We thank the reviewers for their constructive suggestions, particularly the encouragement of the development and inclusion of the explicit relationships given in the appendix.

\section{References}

${ }^{1}$ Riggins, D. W., McClinton, C. R., and Vitt, P. H., "Thrust Losses in Hypersonic Engines Part 1: Methodology," Journal of Propulsion and Power, Vol. 13, No. 2, 1997, pp. 281-287.

${ }^{2}$ Courant, R., and Friedrichs, K. O., Supersonic Flow and Shock Waves, Interscience, New York, 1967, Chap. 3, pp. 204-235.

${ }^{3}$ Foa, J. V., Elements of Flight Propulsion, Wiley, New York, 1960, Chap. 13, pp. 274-287.

${ }^{4}$ Kailasanath, K., "Review of Propulsion Applications of Detonation Waves," AIAA Journal, Vol. 38, No. 9, 2000, pp. 1698-1708.

${ }^{5}$ Heiser, W. H., and Pratt, D. T., "Thermodynamic Cycle Analysis of Pulse Detonation Engines," Journal of Propulsion and Power, Vol. 18, No. 1, 2002, pp. 68-76.

${ }^{6}$ Dunlap, R., Brehm, R. L., and Nicholls, J. A., "A Preliminary Study of the Application of Steady-State Detonative Combustion to a Reaction Engine," Jet Propulsion, Vol. 28, No. 7, 1958, pp. 451-456.

${ }^{7}$ Sargent, W. H., and Gross, R. A., "Detonation Wave Hypersonic Ramjet,"
ARS Journal, Vol. 30, No. 6, 1960, pp. 543-549.

${ }^{8}$ Wintenberger, E., and Shepherd, J. E., "The Performance of Steady Detonation Engines," AIAA Paper 2003-0714, Jan. 2003.

${ }^{9}$ Zel'dovich, Y. B., "On the Use of Detonative Combustion in Power Engineering," Journal of Technical Physics, Vol. 10, No. 17, 1940, pp. 1453-1461 (in Russian).

${ }^{10}$ Foa, J. V., "Single Flow Jet Engines-A Generalized Treatment," Journal of the American Rocket Society, Vol. 21, No. 5, Sept. 1951, pp. 115-126.

${ }^{11}$ Wintenberger, E., and Shepherd, J. E., "Thermodynamic Analysis of Combustion Processes for Propulsion Systems," AIAA Paper 2004-1033, Jan. 2004.

${ }^{12}$ Fickett, W., and Davis, W. C., Detonation Theory and Experiment, Dover, Mineola, NY, 2001, pp. 35-38.

${ }^{13}$ Wintenberger, E., "Application of Steady and Unsteady Detonation Waves to Propulsion," Ph.D. Dissertation, Aeronautics Dept., California Inst. of Technology, Pasadena, CA, June 2004.

${ }^{14}$ Thompson, P. A., Compressible Fluid Dynamics, Advanced Engineering Series, Rensselaer Polytechnic Inst., Troy, NY, 1988, pp. 347-359.

${ }^{15}$ Clarke, J. M., and Horlock, J. H., "Availability and Propulsion," Journal of Mechanical Engineering Science, Vol. 17, No. 4, 1975, pp. 223-232.

${ }^{16}$ Oates, G. C., Aerothermodynamics of Gas Turbines and Rocket Propulsion, AIAA Education Series, AIAA, New York, 1984, Chap. 2, p. 44.

${ }^{17}$ Reynolds, W., "The Element Potential Method for Chemical Equilibrium Analysis: Implementation in the Interactive Program STANJAN," Mechanical Engineering Dept., Tech. Rept., Stanford Univ., Stanford, CA, Jan. 1986. 